\title{
On a non-autonomous stochastic Lotka-Volterra competitive system
}

\author{
Meiling Deng \\ School of Mathematical Science, Huaiyin Normal University, Huaian 223300, P. R. China. \\ Communicated by A. Atangana
}

\begin{abstract}
In this paper, we consider a general non-autonomous Lotka-Volterra competitive model with random perturbations. Sufficient conditions for stochastic permanence and extinction are established. Particularly, when these conditions are applied to a stochastic logistic equation, these conditions are sufficient and necessary. Some figures are also worked out to illustrate the main results. Some recent results are extended. Moreover, our results reveal that different types of stochastic noises have different effects on the permanence and extinction of the population. (C)2017 All rights reserved.
\end{abstract}

Keywords: Competitive system, random perturbations, permanence, extinction. 2010 MSC: 92D25, 60H10, 60H30.

\section{Introduction}

In the natural world, it is a usual phenomena that several species compete for the limited resources. Therefore it is important to study the multi-species competitive models. A famous non-autonomous Lotka-Volterra competitive system can be expressed as follows

$$
\frac{d x_{i}(t)}{d t}=x_{i}(t)\left[r_{i}(t)-\sum_{j=1}^{n} a_{i j}(t) x_{j}(t)\right], \quad i=1, \ldots, n,
$$

where $x_{i}(t)$ is the size of the ith population at time $t, r_{i}(t)$ is the growth rate of the $i$ th species at time $t, a_{i i}(t)>0$ is the introspecific competition rate, and $a_{i j}(t)>0$ is the interspecific competition rate, $i, j=1,2, \ldots, n, i \neq j$. Owing to its theoretical and practical significance, system (1.1) has been extensively investigated and many important properties of the global dynamics of solutions have been obtained. For example, persistence and extinction of (1.1) were considered in [1, 2, 35, 37]. Zhao et al. [38] investigated permanence and global attractivity of model (1.1). Model (1.1) with time delay was analyzed in [13, 17]. $[3,15]$ studied the effect of impulses on model (1.1). Model (1.1) with stage structure was considered by [24].

On the other hand, in the real world, population systems are inevitably affected by environmental noises. Then it is important to study stochastic population systems to reveal the effect of random noise

Email address: hnudengmeiling@163.com (Meiling Deng)

doi:10.22436/jnsa.010.06.24 
on the dynamics of the system. In fact, stochastic population models have been received great attention recently, see e.g. $[4,6-12,16,18-23,25-30,32,33,36,39,40]$. Particularly, under the assumption that the growth rate $r_{i}(t)$ is affected by random noise, with

$$
r_{i}(t) \rightarrow r_{i}(t)+\beta_{i}(t) \dot{W}_{1}(t),
$$

where $\dot{W}_{1}(t)$ stands for the white noise, Li and Mao [18] proposed and investigated the following stochastic competitive model

$$
d x_{i}(t)=x_{i}(t)\left(r_{i}(t)-\sum_{j=1}^{n} a_{i j}(t) x_{j}(t)\right) d t+\beta_{i}(t) x_{i}(t) d W_{1}(t), i=1, \ldots, n,
$$

where $W_{1}(t)$ is a standard Brownian motions defined on a complete probability space $(\Omega, \mathcal{F}, P)$. The authors [18] investigated stochastic permanence, extinction, and global attractivity of model (1.2). From then on, model (1.2) and its generalizations have been investigated by many authors. Bao et al. [7] investigated model (1.2) with Lévy jumps. Jiang et al. [16] considered persistence and extinction of model (1.2) in autonomous case.

However, model (1.2) is based on the assumption that only the growth rates $r_{i}$ are affected by the stochastic noise. Then an important and interesting topic arises naturally: what happens if both $r_{i}$ and $a_{i j}$ are perturbed by the stochastic noise? As a matter of fact, Bandyopadhyay and Chattopadhyay [6] has pointed out that if the parameters in the models are assumed to be deterministic irrespective of environmental fluctuations, there would be some limitations in mathematical modeling of ecological systems, at the same time, it would be difficult to fit data perfectly and to predict the future dynamics of the system accurately. May [34] has claimed that due to environmental noise, the birth rate, competition coefficients, and other parameters in the system should be stochastic. Motivated by these, in this paper, we assume that $a_{i j}$ is also perturbed stochastic noise, with

$$
-a_{i j}(t)+\alpha_{i j}(t) \dot{W}_{2}(t)
$$

Then model (1.2) becomes

$$
\begin{aligned}
d x_{i}(t)= & x_{i}(t)\left(r_{i}(t)-\sum_{j=1}^{n} a_{i j}(t) x_{j}(t)\right) d t \\
& +\beta_{i}(t) x_{i}(t) d W_{1}(t)+\sum_{j=1}^{n} \alpha_{i j}(t) x_{i}(t) x_{j}(t) d W_{2}(t), i=1, \ldots, n,
\end{aligned}
$$

where $W_{1}(t)$ and $W_{2}(t)$ are independent standard Brownian motions defined on a complete probability space $(\Omega, \mathcal{F}, P) ; r_{i}(t), a_{i j}(t), \beta_{i}(t)$, and $\alpha_{i j}(t)$ are continuous and bounded functions on $[0,+\infty)$ and $a_{i j}(t) \geqslant 0$ for $i, j=1, \ldots, n$.

In the investigation of population models, permanence and extinction are two important topics. However, as far as we know, no results related to permanence and extinction of model (1.3) have been reported. The aim of this paper is to study these problems. We will show that when the noise is small, the population system is stochastically permanent. At the same time, we will prove that a sufficiently large noise will force all the populations become extinct. In particular, we shall establish the sufficient and necessary conditions for stochastic permanence and extinction to a general stochastic logistic equation. Some recent results will be generalized. Moreover, we will show that a different type of stochastic noise has a different effect on the permanence and extinction of the population.

\section{Main results}

For the sake of simplicity, define

$$
R_{+}^{n}=\left\{a=\left(a_{1}, . ., a_{n}\right): a_{i}>0,1 \leqslant i \leqslant n\right\}, \quad f^{u}=\sup _{t \geqslant 0} f(t), \quad f^{l}=\inf _{t \geqslant 0} f(t) .
$$


Lemma 2.1. If $\min _{1 \leqslant i, j \leqslant n} a_{i j}^{l}>0$, then for any given initial value $x(0) \in R_{+}^{n}$, there is a unique solution $x(t)$ to (1.3) on $\mathrm{t} \geqslant 0$ and the solution will remain in $\mathrm{R}_{+}^{\mathrm{n}}$ almost surely (a.s.).

Proof. The proof is a slight modification of in [18] and therefore is omitted.

From now on, we always suppose that $\min _{1 \leqslant i, j \leqslant n} a_{i j}^{l}>0$.

Definition 2.2 ([18]). If for all $\varepsilon \in(0,1)$, there exists a pair of positive constants $\xi_{1}=\xi_{1}(\varepsilon)$ and $\xi_{2}=\xi_{2}(\varepsilon)$ such that for any initial data $x(0) \in R_{+}^{n}$, the solution satisfies

$$
\liminf _{t \rightarrow+\infty} P\left\{|x(t)| \geqslant \xi_{1}\right\} \geqslant 1-\varepsilon, \liminf _{t \rightarrow+\infty} P\left\{|x(t)| \leqslant \xi_{2}\right\} \geqslant 1-\varepsilon
$$

then model (1.3) is said to be stochastically permanent.

Lemma 2.3. Suppose that $\mathrm{x}(\mathrm{t})$ is an arbitrary solution of (1.3), then for every $0<\mathrm{p}<1, \boldsymbol{i}=1,2 \ldots, \mathrm{n}$, there is a constant $\mathrm{K}=\mathrm{K}(\mathrm{p})>0$ such that

$$
\limsup _{t \rightarrow+\infty} E\left[x_{i}^{p}(t)\right] \leqslant K(p), t \geqslant 0 .
$$

Proof. The proof is standard but for the completeness of the paper we only give a brief one. Define

$$
V(x)=\sum_{i=1}^{n} e^{t} x_{i}^{p}
$$

for $x \in R_{+}^{n}$, where $p<1$. Applying Itô's formula ([31]) results in

$$
\begin{aligned}
d V(x)= & e^{t} \sum_{i=1}^{n} x_{i}^{p} d t+e^{t} \sum_{i=1}^{n}\left\{p x_{i}^{p-1} d x_{i}+0.5 p(p-1) x_{i}^{p-2}\left(d x_{i}\right)^{2}\right\} \\
= & e^{t} p \sum_{i=1}^{n} x_{i}^{p}\left[1 / p+r_{i}(t)-\sum_{j=1}^{n} a_{i j}(t) x_{j}+0.5(p-1) \beta_{i}^{2}(t)\right. \\
& \left.+0.5(p-1)\left(\sum_{j=1}^{n} \alpha_{i j}(t) x_{j}\right)^{2}\right] d t+e^{t} p \sum_{i=1}^{n} \beta_{i}(t) x_{i}^{p} d W_{1}(t)+e^{t} p \sum_{i=1}^{n} \sum_{j=1}^{n} \alpha_{i j}(t) x_{i}^{p} x_{j} d W_{2}(t) . \\
\leqslant & e^{t} p \sum_{i=1}^{n} x_{i}^{p}\left[1 / p+r_{i}(t)-a_{i i}(t) x_{i}\right] d t+e^{t} p \sum_{i=1}^{n} \beta_{i}(t) x_{i}^{p} d W_{1}(t)+e^{t} p \sum_{i=1}^{n} \sum_{j=1}^{n} \alpha_{i j}(t) x_{i}^{p} x_{j} d W_{2}(t) . \\
\leqslant & e^{t} \sum_{i=1}^{n} K_{i}(p) d t+e^{t} p \sum_{i=1}^{n} \beta_{i}(t) x_{i}^{p} d W_{1}(t)+e^{t} p \sum_{i=1}^{n} \sum_{j=1}^{n} \alpha_{i j}(t) x_{i}^{p} x_{j} d W_{2}(t),
\end{aligned}
$$

where

$$
K_{i}(p)=\left(\frac{p}{p+1}\right)^{p+1}\left(\frac{1 / p+\left|r_{i}\right|^{u}}{a_{i i}^{l}}\right)^{p}
$$

In other words, we have shown that

$$
e^{t} E\left[\sum_{i=1}^{n} x_{i}^{p}(t)\right] \leqslant \sum_{i=1}^{n} x_{i}^{p}(0)+E \int_{0}^{t} e^{s} \sum_{i=1}^{n} K_{i}(p) d s=\sum_{i=1}^{n} x_{i}^{p}(0)+\sum_{i=1}^{n} K_{i}(p)\left(e^{t}-1\right) .
$$

Consequently

$$
\limsup _{t \rightarrow+\infty} E\left[\sum_{i=1}^{n} x_{i}^{p}(t)\right] \leqslant \sum_{i=1}^{n} K_{i}(p)=: K(p)
$$

This completes the proof. 
Now, let us impose an assumption.

(H1): $\min _{1 \leqslant i \leqslant n} b_{i}^{l}>0$, where $b_{i}(t)=r_{i}(t)-0.5 \beta_{i}^{2}(t), 1 \leqslant i \leqslant n$. That is to say, the intensities of the stochastic noises in the system are not too large.

Theorem 2.4. If (H1) is satisfied, then (1.3) is stochastically permanent.

Proof. Let

$$
\mathrm{u}(\mathrm{x})=\sum_{i=1}^{\mathrm{n}} \mathrm{x}_{\mathrm{i}}, \quad \mathrm{v}_{1}(\mathrm{x})=1 / \mathrm{u}^{2}(\mathrm{x})
$$

for $x \in R_{+}^{n}$. It then follows from Itô's formula that

$$
\begin{aligned}
d V_{1}(x)= & -\frac{2}{u^{3}(x)} \sum_{i=1}^{n} x_{i}\left(r_{i}(t)-\sum_{j=1}^{n} a_{i j}(t) x_{j}\right) d t+\frac{3}{U^{4}(x)}\left[\left(\sum_{i=1}^{n} \beta_{i}(t) x_{i}\right)^{2}+\left(\sum_{i=1}^{n} \sum_{j=1}^{n} \alpha_{i j}^{2}(t) x_{i} x_{j}\right)^{2}\right] d t \\
& -\frac{2}{U^{3}(x)}\left[\sum_{i=1}^{n} \beta_{i}(t) x_{i} d W_{1}(t)+\sum_{i=1}^{n} \sum_{j=1}^{n} \alpha_{i j}(t) x_{i} x_{j} d W_{2}(t)\right] .
\end{aligned}
$$

By (H1), there is a positive constant $\lambda$ satisfying

$$
\min _{1 \leqslant i \leqslant n} b_{i}^{l}>\lambda \max _{1 \leqslant i \leqslant n}\left(\beta_{i}^{2}\right)^{u} .
$$

Let

$$
V_{2}(x)=\left(1+V_{1}(x)\right)^{\lambda}, \quad x \in R_{+}^{n}
$$

Then

$$
d V_{2}(x)=L V_{2}(x) d t-\lambda\left(1+V_{1}(x)\right)^{\lambda-1} \frac{2}{U^{3}(x)} \times\left[\sum_{i=1}^{n} \beta_{i}(t) x_{i} d W_{1}(t)+\sum_{i=1}^{n} \sum_{j=1}^{n} \alpha_{i j}(t) x_{i} x_{j} d W_{2}(t)\right],
$$

where

$$
\begin{aligned}
& \operatorname{LV}_{2}(x)=\lambda\left(1+V_{1}(x)\right)^{\lambda-2}\left\{-\left(1+V_{1}(x)\right) \frac{2}{U^{3}(x)} \sum_{i=1}^{n} x_{i}\left(r_{i}(t)-\sum_{j=1}^{n} a_{i j}(t) x_{j}\right)\right. \\
& +\left(1+V_{1}(x)\right) \frac{3}{U^{4}(x)}\left[\sum_{i=1}^{n} \beta_{i}^{2}(t) x_{i}^{2}+\sum_{i=1}^{n} \sum_{j=1}^{n} \alpha_{i j}^{2}(t) x_{i}^{2} x_{j}^{2}\right] \\
& \left.+\frac{\lambda-1}{2} \frac{4}{\mathrm{u}^{6}(x)}\left[\left(\sum_{i=1}^{n} \beta_{i}(t) x_{i}\right)^{2}+\left(\sum_{i=1}^{n} \sum_{j=1}^{n} \alpha_{i j}(t) x_{i} x_{j}\right)^{2}\right]\right\} \\
& =\lambda\left(1+v_{1}(x)\right)^{\lambda-2}\left\{-\frac{2}{u^{3}(x)} \sum_{i=1}^{n} x_{i} r_{i}(t)-\frac{2}{u^{5}(x)} \sum_{i=1}^{n} x_{i} r_{i}(t)\right. \\
& +\frac{2}{\mathrm{u}^{3}(\mathrm{x})} \sum_{i=1}^{n} \sum_{j=1}^{n} a_{i j}(t) x_{i} x_{j}+\frac{2}{\mathrm{u}^{5}(\mathrm{x})} \sum_{i=1}^{n} \sum_{j=1}^{n} a_{i j}(t) x_{i} x_{j} \\
& \left.+\left[\frac{3}{u^{4}(x)}+\frac{3}{u^{6}(x)}+\frac{2(\lambda-1)}{u^{6}(x)}\right]\left[\left(\sum_{i=1}^{n} \beta_{i}(t) x_{i}\right)^{2}+\left(\sum_{i=1}^{n} \sum_{j=1}^{n} \alpha_{i j}(t) x_{i} x_{j}\right)^{2}\right]\right\} \\
& \leqslant \lambda\left(1+V_{1}(x)\right)^{\lambda-2}\left\{-\frac{2}{u^{6}(x)} \sum_{i=1}^{n} x_{i}^{2} r_{i}(t)+\frac{\max _{1 \leqslant i, j \leqslant n} a_{i j}^{\mathfrak{u}}}{u(x)}+\frac{\max _{1 \leqslant i, j \leqslant n} a_{i j}^{\mathfrak{u}}}{u^{3}(x)}\right.
\end{aligned}
$$




$$
\begin{aligned}
& \left.+\frac{3 \max _{1 \leqslant i \leqslant n}\left(\beta_{i}^{2}\right)^{u}}{u^{2}(x)}+3 \max _{1 \leqslant i, j \leqslant n}\left(\alpha_{i j}^{2}\right)^{u}+\frac{2 \lambda+1}{u^{6}(x)} \sum_{i=1}^{n} \beta_{i}^{2}(t) x_{i}^{2}+\frac{(2 \lambda+1) \max _{1 \leqslant i, j \leqslant n}\left(\alpha_{i j}^{2}\right)^{\mathfrak{u}}}{u^{2}(x)}\right\} \\
= & \lambda\left(1+V_{1}(x)\right)^{\lambda-2}\left\{-\frac{2}{u^{6}(x)} \sum_{i=1}^{n} x_{i}^{2}\left[b_{i}(t)-\lambda \beta_{i}^{2}(t)\right]+V_{1}^{1.5}(x) \max _{1 \leqslant i, j \leqslant n} a_{i j}^{u}\right. \\
& \left.+V_{1}(x)\left[3 \max _{1 \leqslant i \leqslant n}\left(\beta_{i}^{2}\right)^{u}+(2 \lambda+1) \max _{1 \leqslant i, j \leqslant n}\left(\alpha_{i j}^{2}\right)^{u}\right]+V_{1}^{0.5}(x) \max _{1 \leqslant i, j \leqslant n} a_{i j}^{u}+3 \max _{1 \leqslant i, j \leqslant n}\left(\alpha_{i j}^{2}\right)^{u}\right\} \\
\leqslant & \lambda\left(1+V_{1}(x)\right)^{\lambda-2}\left\{-2 V_{1}^{2}(x)\left[\min _{1 \leqslant i \leqslant n} b_{i}^{\mathfrak{l}}-\lambda \max _{1 \leqslant i \leqslant n}\left(\beta_{i}^{2}\right)^{\mathfrak{u}}\right]+V_{1}^{1.5}(x) \max _{1 \leqslant i, j \leqslant n} a_{i j}^{u}\right. \\
& \left.+V_{1}(x)\left[3 \max _{1 \leqslant i \leqslant n}\left(\beta_{i}^{2}\right)^{u}+(2 \lambda+1) \max _{1 \leqslant i, j \leqslant n}\left(\alpha_{i j}^{2}\right)^{u}\right]+V_{1}^{0.5}(x) \max _{1 \leqslant i, j \leqslant n} a_{i j}^{u}+3 \max _{1 \leqslant i, j \leqslant n}\left(\alpha_{i j}^{2}\right)^{u}\right\} .
\end{aligned}
$$

Let $v$ be sufficiently small such that

$$
0<0.5 v / \lambda<\min _{1 \leqslant i \leqslant n} b_{i}^{\mathfrak{l}}-\lambda \max _{1 \leqslant i \leqslant n}\left(\alpha_{i}^{2}\right)^{\mathfrak{u}} .
$$

Let

$$
V_{3}(x(t))=e^{v t} V_{2}(x(t))
$$

An application of Itô's formula, gives

$$
\begin{aligned}
d V_{3}(x) & =v e^{v t} V_{2}(x) d t+e^{v t} d V_{2}(x) \\
& =L V_{3}(x) d t-\lambda e^{v t}\left(1+V_{1}(x)\right)^{\lambda-1} \frac{2}{U^{3}(x)} \times\left[\sum_{i=1}^{n} \beta_{i}(t) x_{i} d W_{1}(t)+\sum_{i=1}^{n} \sum_{j=1}^{n} \alpha_{i j}(t) x_{i} x_{j} d W_{2}(t)\right],
\end{aligned}
$$

where

$$
\begin{aligned}
\operatorname{LV}_{3}(x) \leqslant & \lambda e^{v \mathrm{t}}\left(1+\mathrm{V}_{1}(x)\right)^{\lambda-2}\left\{v\left(1+V_{1}(x)\right)^{2} / \lambda-2 V_{1}^{2}(x)\left[\min _{1 \leqslant i \leqslant n} b_{i}^{\mathrm{l}}-\lambda \max _{1 \leqslant i \leqslant n}\left(\beta_{i}^{2}\right)^{u}\right]\right. \\
& +V_{1}^{1.5}(x) \max _{1 \leqslant i, j \leqslant n} a_{i j}^{\mathfrak{u}}+V_{1}(x)\left[3 \max _{1 \leqslant i \leqslant n}\left(\beta_{i}^{2}\right)^{u}+(2 \lambda+1) \max _{1 \leqslant i, j \leqslant n}\left(\alpha_{i j}^{2}\right)^{u}\right] \\
& \left.+V_{1}^{0.5}(x) \max _{1 \leqslant i, j \leqslant n} a_{i j}^{\mathfrak{u}}+3 \max _{1 \leqslant i, j \leqslant n}\left(\alpha_{i j}^{2}\right)^{u}\right\} \\
= & \lambda e^{v t}\left(1+V_{1}(x)\right)^{\lambda-2}\left\{-2 V_{1}^{2}(x)\left[\min _{1 \leqslant i \leqslant n} b_{i}^{\mathfrak{l}}-\lambda \max _{1 \leqslant i \leqslant n}\left(\beta_{i}^{2}\right)^{u}-0.5 v / \lambda\right]\right. \\
& +V_{1}^{1.5}(x) \max _{1 \leqslant i, j \leqslant n} a_{i j}^{u}+V_{1}(x)\left[3 \max _{1 \leqslant i \leqslant n}\left(\beta_{i}^{2}\right)^{u}+(2 \lambda+1) \max _{1 \leqslant i, j \leqslant n}\left(\alpha_{i j}^{2}\right)^{u}+2 v / \lambda\right] \\
& \left.+V_{1}^{0.5}(x) \max _{1 \leqslant i, j \leqslant n} a_{i j}^{u}+3 \max _{1 \leqslant i, j \leqslant n}\left(\alpha_{i j}^{2}\right)^{u}+v / \lambda\right\}=e^{v t} J(x),
\end{aligned}
$$

where

$$
\begin{aligned}
J(x)= & \lambda\left(1+V_{1}(x)\right)^{\lambda-2}\left\{-2 V_{1}^{2}(x)\left[\min _{1 \leqslant i \leqslant n} b_{i}^{\mathfrak{l}}-\lambda \max _{1 \leqslant i \leqslant n}\left(\beta_{i}^{2}\right)^{u}-0.5 v / \lambda\right]\right. \\
& +V_{1}^{1.5}(x) \max _{1 \leqslant i, j \leqslant n} a_{i j}^{u}+V_{1}(x)\left[3 \max _{1 \leqslant i \leqslant n}\left(\beta_{i}^{2}\right)^{u}+(2 \lambda+1) \max _{1 \leqslant i, j \leqslant n}\left(\alpha_{i j}^{2}\right)^{u}+2 v / \lambda\right] \\
& \left.+V_{1}^{0.5}(x) \max _{1 \leqslant i, j \leqslant n} a_{i j}^{u}+3 \max _{1 \leqslant i, j \leqslant n}\left(\alpha_{i j}^{2}\right)^{u}+v / \lambda\right\} .
\end{aligned}
$$

Clearly, $J(x)$ has an upper bound in $R_{+}^{n}$, so we define $C_{1}:=\sup _{x \in R_{+}^{n}} J(x)<+\infty$. Consequently,

$$
d V_{3}(x(t)) \leqslant C_{1} e^{v t} d t-\lambda e^{v t}\left(1+V_{1}(x)\right)^{\lambda-1} \frac{2}{U^{3}(x)}\left[\sum_{i=1}^{n} \beta_{i}(t) x_{i} d W_{i}(t)+\sum_{i=1}^{n} \sum_{j=1}^{n} \alpha_{i j}(t) x_{i} x_{j} d W_{2}(t)\right]
$$


Integrating both sides of the above inequality and then taking expectations, we have

$$
E\left[V_{3}(x(t))\right]=E\left[e^{v t}\left(1+V_{1}(x(t))\right)^{\lambda}\right] \leqslant\left(1+V_{1}(x(0))\right)^{\lambda}+C_{1} e^{v t} / v
$$

That is to say,

$$
\limsup _{t \rightarrow+\infty} E\left[V_{1}^{\lambda}(x(t))\right] \leqslant C_{1} / v
$$

Thanks to

$$
\left(\sum_{i=1}^{n} x_{i}(t)\right)^{\lambda} \leqslant\left(n \max _{1 \leqslant i \leqslant n} x_{i}(t)\right)^{\lambda}=n^{\lambda}\left(\max _{1 \leqslant i \leqslant n} x_{i}^{2}(t)\right)^{0.5 \lambda} \leqslant n^{\lambda}|x(t)|^{\lambda}
$$

Then we have

$$
\limsup _{t \rightarrow+\infty} E\left[|x(t)|^{-2 \lambda}\right] \leqslant n^{2 \lambda} C_{1} / v=: C .
$$

For arbitrary fixed $\varepsilon>0$, denote $\xi_{1}=\varepsilon^{0.5 / \lambda} / \mathrm{C}^{0.5 / \lambda}$, according to Chebyshev's inequality ([31]), one can see that

$$
\mathrm{P}\left\{|x(\mathrm{t})|<\xi_{1}\right\} \leqslant \xi_{1}^{2 \lambda} E\left[1 /|x(\mathrm{t})|^{2 \lambda}\right] .
$$

Consequently, $\liminf _{t \rightarrow+\infty} P\left\{|x(t)| \geqslant \xi_{1}\right\} \geqslant 1-\varepsilon$.

To complete the proof, it suffices to prove that for arbitrary fixed $\varepsilon>0$, we can find a constant $\xi_{2}>0$ such that $\liminf _{t \rightarrow+\infty} P\left\{|x(t)| \leqslant \xi_{2}\right\} \geqslant 1-\varepsilon$. In fact, by (2.1) and Chebyshev's inequality, one can prove this assertion easily.

We are in the position to study the extinction of (1.3).

Theorem 2.5. If $\limsup _{t \rightarrow+\infty} t^{-1} \int_{0}^{t} b_{i}(s) d s<0$, then the population $x_{i}(t)$, represented by model (1.3) goes to extinction a.s., i.e., $\lim _{\mathrm{t} \rightarrow+\infty} \mathrm{x}_{\mathrm{i}}(\mathrm{t})=0$ a.s., $1 \leqslant i \leqslant n$.

Proof. By virtue of Itô's formula,

$$
d \ln x_{i}=\left[b_{i}(t)-\sum_{j=1}^{n} a_{i j}(t) x_{i}-0.5\left(\sum_{j=1}^{n} \alpha_{i j}(t) x_{j}\right)^{2}\right] d t+\beta_{i}(t) d W_{1}(t)+\sum_{j=1}^{n} \alpha_{i j}(t) x_{j} d W_{2}(t), \quad 1 \leqslant i \leqslant n .
$$

Integrating both sides from 0 to $t$,

$$
\begin{aligned}
\ln x_{i}(t)-\ln x_{i}(0)= & \int_{0}^{t}\left[b_{i}(s)-\sum_{j=1}^{n} a_{i j}(s) x_{i}(s)-0.5\left(\sum_{j=1}^{n} \alpha_{i j}(t) x_{j}(s)\right)^{2}\right] d s \\
& +M_{i}(t)+N_{i}(t), \quad 1 \leqslant i \leqslant n .
\end{aligned}
$$

Here

$$
M_{i}(t)=\int_{0}^{t} \beta_{i}(s) d W_{1}(s), N_{i}(t)=\int_{0}^{t} \sum_{j=1}^{n} \alpha_{i j}(s) x_{j}(s) d W_{2}(s), \quad 1 \leqslant i \leqslant n .
$$

Clearly, $M_{i}(t)$ is a local martingale with quadratic variation

$$
\left\langle M_{i}(t), M_{i}(t)\right\rangle=\int_{0}^{t} \beta_{i}^{2}(s) d s \leqslant\left(\beta_{i}^{2}\right)^{u} t .
$$


It then follows from the strong law of large numbers for local martingales (see, e.g., [31]) that

$$
\lim _{t \rightarrow+\infty} M_{i}(t) / t=0 \quad \text { a.s., } 1 \leqslant i \leqslant n .
$$

At the same time,

$$
\left\langle N_{i}(t), N_{i}(t)\right\rangle=\int_{0}^{t}\left(\sum_{j=1}^{n} \alpha_{i j}(s) x_{j}(s)\right)^{2} d s .
$$

According to the exponential martingale inequality (see, e.g., [31]), one can observe that

$$
P\left\{\sup _{0 \leqslant t \leqslant k}\left[N_{i}(t)-\frac{1}{2}\left\langle N_{i}(t), N_{i}(t)\right\rangle\right]>2 \ln k\right\} \leqslant 1 / k^{2}, \quad 1 \leqslant i \leqslant n .
$$

Thanks to the Borel-Cantalli lemma (see, e.g., [31]), for almost all $\omega \in \Omega$, there exists a random integer $k_{0}=k_{0}(\omega)$ such that for $k \geqslant k_{0}$,

$$
\sup _{0 \leqslant t \leqslant k}\left[N_{i}(t)-\frac{1}{2}\left\langle N_{i}(t), N_{i}(t)\right\rangle\right] \leqslant 2 \ln k, 1 \leqslant i \leqslant n .
$$

Therefore,

$$
N_{i}(t) \leqslant 2 \ln k+0.5 \int_{0}^{t}\left(\sum_{j=1}^{n} \alpha_{i j}(s) x_{j}(s)\right)^{2} d s
$$

for all $0 \leqslant t \leqslant k, k \geqslant k_{0}$ almost surely. When these inequalities are used in (2.2), we have

$$
\begin{aligned}
\ln x_{i}(t)-\ln x_{i}(0)= & \int_{0}^{t}\left[b_{i}(s)-\sum_{j=1}^{n} a_{i j}(s) x_{i}(s)-0.5\left(\sum_{j=1}^{n} \alpha_{i j}(s) x_{j}(s)\right)^{2}\right] d s \\
& +M_{i}(t)+2 \ln k+0.5 \int_{0}^{t}\left(\sum_{j=1}^{n} \alpha_{i j}(s) x_{j}(s)\right)^{2} d s \\
\leqslant & \int_{0}^{t} b_{i}(s) d s+M_{i}(t)+2 \ln k, \quad 1 \leqslant i \leqslant n
\end{aligned}
$$

for all $0 \leqslant t \leqslant k, k \geqslant k_{0}$ almost surely. That is to say, for $0<k-1 \leqslant t \leqslant k$,

$$
t^{-1}\left[\ln x_{i}(t)-\ln x_{i}(0)\right] \leqslant t^{-1} \int_{0}^{t} b_{i}(s) d s+\frac{2 \ln k}{t}+M_{i}(t) / t \leqslant t^{-1} \int_{0}^{t} b_{i}(s) d s+\frac{2 n \ln k}{k-1}+M_{i}(t) / t .
$$

According to (2.3),

$$
\limsup _{t \rightarrow+\infty} t^{-1} \ln x_{i}(t) \leqslant \limsup _{t \rightarrow+\infty} t^{-1} \int_{0}^{t} b_{i}(s) d s .
$$

That is to say, if $\limsup _{t \rightarrow+\infty} t^{-1} \int_{0}^{t} b_{i}(s) d s<0$, then $\lim _{t \rightarrow+\infty} x_{i}(t)=0$.

Remark 2.6. Biologically, Theorem 2.5 means that sufficiently large stochastic random noises can force the species become extinct.

Remark 2.7. Li and Mao [18] have studied model (1.2) and have shown that

(A) if (H1) is satisfied, then (1.2) is stochastically permanent;

(B) if $\limsup _{t \rightarrow+\infty} t^{-1} \int_{0}^{t} b_{i}(s) d s<0$, then $x_{i}(t)$, represented by model (1.2), goes to extinction a.s., i.e., $\lim _{\mathrm{t} \rightarrow+\infty} x_{i}(t)=0$ a.s., $1 \leqslant i \leqslant n$.

Clearly, model (1.2) is a special case of model (1.3) (i.e., $\alpha_{i j}(t) \equiv 0$ for all $1 \leqslant i, j \leqslant n$ ). Therefore our Theorems 2.4 and 2.5 extend the results of [18]. 
To finish this section, we consider the following stochastic logistic equation:

$$
d x(t)=x(t)(r-a x(t)) d t+\beta x(t) d W_{1}(t)+\alpha x^{2} d W_{2}(t), \quad x(0)=x_{0}>0,
$$

where $a>0$. By Theorems 2.4 and 2.5, we obtain the following sufficient and necessary conditions for stochastic permanence and extinction.

Corollary 2.8. For Eq. (2.4):

(I) if $r-0.5 \beta^{2}<0$, then the population $x(t)$ is extinctive a.s.;

(II) if $\mathrm{r}-0.5 \beta^{2}>0$, then the population $\mathrm{x}(\mathrm{t})$ is stochastically permanent.

Remark 2.9. Corollary 2.8 reveals an interesting and important result: different types of stochastic noises have different effects on the permanence and extinction of the population. Note that the permanence and extinction of species depend only on the value of $r-0.5 \beta^{2}$. Therefore, the stochastic noise on $r$ is unfavorable for the permanence of the population while the stochastic noises on a has no impact on the permanence and extinction of the population.

\section{Example and numerical simulations}

In this section we use the Milstein method (see, e.g., Higham [14]) to substantiate our main results. For simplicity, we choose $n=2$.

Consider the discretization equations:

$$
\begin{aligned}
x_{1}^{(k+1)}= & x_{1}^{(k)}+x_{1}^{(k)}\left[r_{1}(k \Delta t)-a_{11}(k \Delta t) x_{1}^{(k)}-a_{12}(k \Delta t) x_{2}^{(k)}\right] \Delta t \\
& +\beta_{1}(k \Delta t) x_{1}^{(k)} \sqrt{\Delta t} \gamma^{(k)}+\frac{\beta_{1}^{2}(k \Delta t)}{2} x_{1}^{(k)}\left(\left(\gamma^{(k)}\right)^{2}-1\right) \Delta t \\
& +\alpha_{11}(k \Delta t)\left(x_{1}^{(k)}\right)^{2} \sqrt{\Delta t} \eta^{(k)}+\frac{\alpha_{11}^{2}(k \Delta t)}{2}\left(x_{1}^{(k)}\right)^{2}\left(\left(\eta^{(k)}\right)^{2}-1\right) \Delta t \\
& +\alpha_{12}(k \Delta t) x_{1}^{(k)} x_{2}^{(k)} \sqrt{\Delta t} \eta^{(k)}+\frac{\alpha_{12}^{2}(k \Delta t)}{2} x_{1}^{(k)} x_{2}^{(k)}\left(\left(\eta^{(k)}\right)^{2}-1\right) \Delta t \\
x_{2}^{(k+1)}= & x_{2}^{(k)}+x_{2}^{(k)}\left[r_{2}(k \Delta t)-a_{21}(k \Delta t) x^{(k)}-a_{22}(k \Delta t) x_{2}^{(k)}\right] \Delta t \\
& +\beta_{2}(k \Delta t) x_{2}^{(k)} \sqrt{\Delta t} \gamma^{(k)}+\frac{\beta_{2}^{2}(k \Delta t)}{2} x_{2}^{(k)}\left(\left(\gamma^{(k)}\right)^{2}-1\right) \Delta t \\
& +\alpha_{21}(k \Delta t) x_{1}^{(k)} x_{2}^{(k)} \sqrt{\Delta t} \eta^{(k)}+\frac{\alpha_{21}^{2}(k \Delta t)}{2} x_{1}^{(k)} x_{2}^{(k)}\left(\left(\eta^{(k)}\right)^{2}-1\right) \Delta t \\
& +\alpha_{22}(k \Delta t)\left(x_{2}^{(k)}\right)^{2} \sqrt{\Delta t} \eta^{(k)}+\frac{\alpha_{22}^{2}(k \Delta t)}{2}\left(x_{2}^{(k)}\right)^{2}\left(\left(\eta^{(k)}\right)^{2}-1\right) \Delta t,
\end{aligned}
$$

where $\gamma_{k}$ and $\eta_{k}, k=1,2, \ldots, n$, are the Gaussian random variables $N(0,1)$.

In Fig. 1, we choose $r_{1}(t)=0.36+0.01 \sin t, r_{2}(t)=0.33+0.05 \sin t, a_{11}(t)=0.2+0.01 \sin t, a_{22}(t)=$ $0.22+0.01 \sin t, a_{12}(t)=0.1+0.02 \sin t, a_{21}(t)=0.05+0.02 \sin t, \alpha_{11}(t)=0.5+0.2 \sin t, \alpha_{12}(t)=0.3+$ $0.1 \sin t, \alpha_{21}(t)=0.7+0.12 \sin t$, and $\alpha_{22}(t)=0.46+0.3 \sin t$. The only difference between conditions of Fig. 1 (a) and Fig. 1 (b) is that the values of $\beta_{1}^{2}$ and $\beta_{2}^{2}$ are different. In Fig. 1 (a), we choose $\beta_{1}^{2}(t) / 2=\beta_{2}^{2}(t) / 2=0.21+0.02 \sin t$, then (H1) holds. In view of Theorem 2.4, system (1.3) is stochastically permanent. Fig. 1 (a) confirms this. In Fig. $1(b)$, we choose $\beta_{1}^{2}(t) / 2=0.38+0.02 \sin t, \beta_{2}^{2}(t) / 2=$ $0.4+0.02 \sin t$, then $\limsup t^{-1} \int_{0}^{t} b_{1}(s) d s=-0.02<0$ and $\lim \sup ^{-1} \int_{0}^{t} b_{2}(s) d s=-0.07<0$. By virtue of Theorem 2.5, both $x_{1}$ and $x_{2}$ go to extinction. See Fig. 1 (b). 


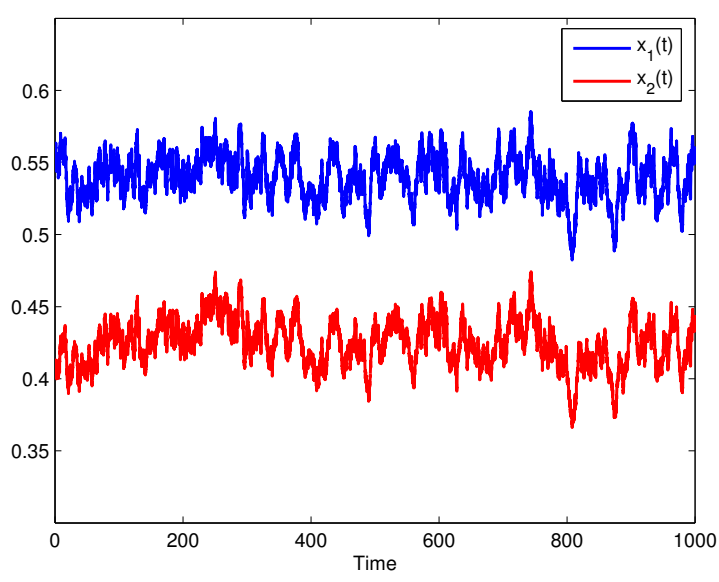

(a)

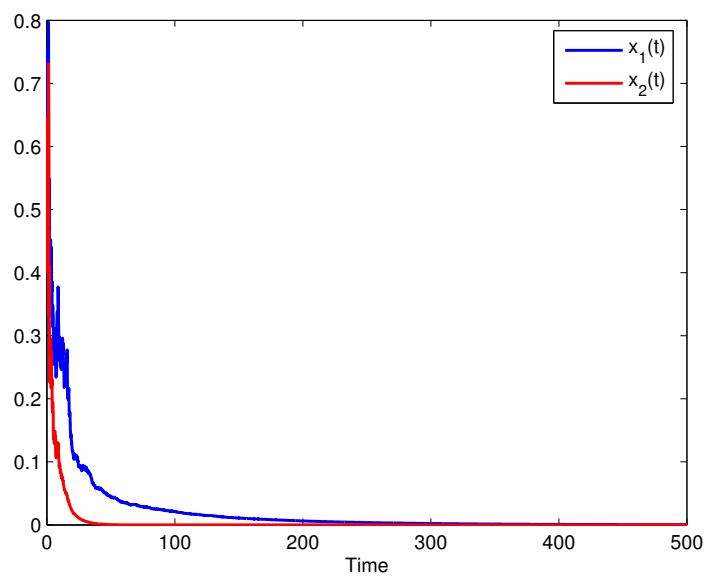

(b)

Figure 1: Solutions of system (1.3) for $n=2, r_{1}(t)=0.36+0.01 \sin t, r_{2}(t)=0.33+0.05 \sin t, a_{11}(t)=0.2+0.01 \sin t, a_{22}(t)=$ $0.22+0.01 \sin t, a_{12}(t)=0.1+0.02 \sin t, a_{21}(t)=0.05+0.02 \sin t, \alpha_{11}(t)=0.5+0.2 \sin t, \alpha_{12}(t)=0.3+0.1 \sin t, \alpha_{21}(t)=$ $0.7+0.12 \sin t$ and $\alpha_{22}(t)=0.46+0.3 \sin t, x_{1}(0)=0.55, x_{2}(0)=0.4$, and step size $\Delta t=0.001$. The horizontal axis represents the time $t$. (a) is with $\beta_{1}^{2}(\mathrm{t}) / 2=\beta_{2}^{2}(\mathrm{t}) / 2=0.21+0.02 \sin \mathrm{t} ;(\mathrm{b})$ is with $\beta_{1}^{2}(\mathrm{t}) / 2=0.38+0.02 \sin \mathrm{t}, \beta_{2}^{2}(\mathrm{t}) / 2=0.4+0.02 \sin \mathrm{t}$.

\section{Conclusions and further research}

This paper is devoted to the permanence and extinction of a general Lotka-Volterra competitive model with random perturbations. We show that when the noise is sufficiently small the population system is stochastically permanent; at the same time, we prove that a sufficiently large noise will force all the populations become extinct. Particularly, for a stochastic logistic equation, sufficient and necessary conditions for stochastic permanence and extinction are established. Some recent results are generalized.

Some interesting topics deserve further investigation. One could study more realistic but more complex models, for example, stochastic systems under regime switching (see e.g., [30, 40]), or with Lévy jumps (see e.g., [29]), or with reaction-diffusion ([5]). Also it is interesting to study n-dimensional stochastic food chain model or cooperative system, and we leave these for future work.

\section{Acknowledgment}

The author thanks the editor and the anonymous referees for their careful reading and valuable comments, which greatly improved the presentation of the paper.

\section{References}

[1] S. Ahmad, On the nonautonomous Volterra-Lotka competition equations, Proc. Amer. Math. Soc., 117 (1993), $199-204$. 1

[2] S. Ahmad, Extinction of species in nonautonomous Lotka-Volterra systems, Proc. Amer. Math. Soc., 127 (1999), $2905-$ 2910. 1

[3] S. Ahmad, I. M. Stamova, Asymptotic stability of an N-dimensional impulsive competitive system, Nonlinear Anal. Real World Appl., 8 (2007), 654-663. 1

[4] A. Bahar, X.-R. Mao, Stochastic delay Lotka-Volterra model, J. Math. Anal. Appl., 292 (2004), 364-380. 1

[5] C.-Z. Bai, Multiplicity of solutions for a class of non-local elliptic operators systems, Bull. Korean Math. Soc., 54 (2017), 715-729. 4

[6] M. Bandyopadhyay, J. Chattopadhyay, Ratio-dependent predator-prey model: effect of environmental fluctuation and stability, Nonlinearity, 18 (2005), 913-936. 1, 1

[7] J.-H. Bao, X.-R. Mao, G. Yin, C.-G. Yuan, Competitive Lotka-Volterra population dynamics with jumps, Nonlinear Anal., 74 (2011), 6601-6616.1 
[8] Y.-L. Cai, Y. Kang, M. Banerjee, W.-M. Wang, A stochastic SIRS epidemic model with infectious force under intervention strategies, J. Differential Equations, 259 (2015), 7463-7502.

[9] Y.-L. Cai, Y. Kang, M. Banerjee, W.-M. Wang, A stochastic epidemic model incorporating media coverage, Commun. Math. Sci., 14 (2016), 892-910.

[10] N. H. Du, V. H. Sam, Dynamics of a stochastic Lotka-Volterra model perturbed by white noise, J. Math. Anal. Appl., 324 (2006), 82-97.

[11] T. C. Gard, Persistence in stochastic food web models, Bull. Math. Biol., 46 (1984), 357-370.

[12] T. C. Gard, Stability for multispecies population models in random environments, Nonlinear Anal., 10 (1986), 1411-1419. 1

[13] K. Gopalsamy, Stability and oscillations in delay differential equations of population dynamics, Mathematics and its Applications, Kluwer Academic Publishers Group, Dordrecht, (1992). 1

[14] D. J. Higham, An algorithmic introduction to numerical simulation of stochastic differential equations, SIAM Rev., 43 (2001), 525-546. 3

[15] J. Hou, Z.-D. Teng, S.-J. Gao, Permanence and global stability for nonautonomous N-species Lotka-Valterra competitive system with impulses, Nonlinear Anal. Real World Appl., 11 (2010), 1882-1896. 1

[16] D.-Q. Jiang, C.-Y. Ji, X.-Y. Li, D. O'Regan, Analysis of autonomous Lotka-Volterra competition systems with random perturbation, J. Math. Anal. Appl., 390 (2012), 582-595. 1, 1

[17] Y. Kuang, Delay differential equations with applications in population dynamics, Mathematics in Science and Engineering, Academic Press, Inc., Boston, MA, (1993). 1

[18] X.-Y. Li, X.-R. Mao, Population dynamical behavior of non-autonomous Lotka-Volterra competitive system with random perturbation, Discrete Contin. Dyn. Syst., 24 (2009), 523-545. 1, 1, 2, 2.2, 2.7

[19] M. Liu, C.-Z. Bai, Optimal harvesting of a stochastic logistic model with time delay, J. Nonlinear Sci., 25 (2015), $277-289$.

[20] M. Liu, C.-Z. Bai, Analysis of a stochastic tri-trophic food-chain model with harvesting, J. Math. Biol., 73 (2016), $597-625$.

[21] M. Liu, C.-Z. Bai, Dynamics of a stochastic one-prey two-predator model with Léry jumps, Appl. Math. Comput., 284 (2016), 308-321.

[22] M. Liu, C.-Z. Bai, Optimal harvesting of a stochastic delay competitive model, Discrete Contin. Dyn. Syst., 22 (2017), 1493-1508.

[23] M. Liu, C.-Z. Bai, Y. Jin, Population dynamical behavior of a two-predator one-prey stochastic model with time delay, Discrete Contin. Dyn. Syst., 37 (2017), 2513-2538. 1

[24] S.-Q. Liu, L.-S. Chen, Z.-J. Liu, Extinction and permanence in nonautonomous competitive system with stage structure, J. Math. Anal. Appl., 274 (2002), 667-684. 1

[25] M. Liu, M. Fan, Permanence of stochastic Lotka-Volterra systems, J. Nonlinear Sci., 27 (2017), 425-452. 1

[26] M. Liu, M. Fan, Stability in distribution of a three-species stochastic cascade predator-prey system with time delays, IMA J. Appl. Math., 82 (2017) 396-423.

[27] M. Liu, K. Wang, Persistence and extinction in stochastic non-autonomous logistic systems, J. Math. Anal. Appl., 375 (2011), 443-457.

[28] M. Liu, K. Wang, Stochastic Lotka-Volterra systems with Lvy noise, J. Math. Anal. Appl., 410 (2014), 750-763.

[29] M. Liu, K. Wang, Q. Wu, Survival analysis of stochastic competitive models in a polluted environment and stochastic competitive exclusion principle, Bull. Math. Biol., 73 (2011), 1969-2012. 4

[30] Q. Luo, X.-R. Mao, Stochastic population dynamics under regime switching, J. Math. Anal. Appl., 334 (2007), 69-84. 1, 4

[31] X.-R. Mao, Stochastic differential equations and their applications, Horwood Publishing Series in Mathematics \& Applications, Horwood Publishing Limited, Chichester, (1997). 2, 2, 2

[32] X.-R. Mao, G. Marion, E. Renshaw, Environmental Brownian noise suppresses explosions in population dynamics, Stochastic Process. Appl., 97 (2002), 95-110. 1

[33] X.-R. Mao, S. Sabanis, E. Renshaw, Asymptotic behaviour of the stochastic Lotka-Volterra model, J. Math. Anal. Appl., 287 (2003), 141-156. 1

[34] R. M. May, Stability and complexity in model ecosystems, Princeton Univ. Press, Princeton, (2001). 1

[35] F. Montesf de Oca, M. L. Zeeman, Extinction in nonautonomous competitive Lotka-Volterra systems, Proc. Amer. Math. Soc., 124 (1996), 3677-3687. 1

[36] S.-L. Pang, F.-Q. Deng, X.-R. Mao, Asymptotic properties of stochastic population dynamics, Dyn. Contin. Discrete Impuls. Syst. Ser. A Math. Anal., 15 (2008), 603-620. 1

[37] Z.-D. Teng, On the non-autonomous Lotka-Volterra N-species competing systems, Appl. Math. Comput., 114 (2000), 175-185. 1

[38] J.-D. Zhao, J.-F. Jiang, A. C. Lazer, The permanence and global attractivity in a nonautonomous Lotka-Volterra system, Nonlinear Anal. Real World Appl., 5 (2004), 265-276. 1

[39] Y. Zhu, M. Liu, Permanence and extinction in a stochastic service-resource mutualism model, Appl. Math. Lett., 69 (2017), 1-7. 1

[40] C. Zhu, G. Yin, On competitive Lotka-Volterra model in random environments, J. Math. Anal. Appl., 357 (2009), $154-170$. 1,4 